\title{
JUSTICIA CIVIL Y TECNOLOGÍAS DE LA INFORMACIÓN Y LAS COMUNICACIONES (TIC) EN ESPAÑA
}

\author{
María Concepción Rayón Ballesteros*
}

\begin{abstract}
This chapter presents how it is carrying out the modernization of the Administration of Justice in Spain, especially in the field of civil procedure with written submissions electronically and the management and processing platform "Lexnet".

Key words: Modernization of the Justice Administration in Spain, computerization, information and communications technology (ICT), electronic documents, Administration of Justice in Spain, Lexnet.
\end{abstract}

\section{EL NECESARIO AVANCE EN LA MODERNIZACIÓN DE LA ADMINISTRACIÓN DE JUSTICIA}

Hoy en día el avance de las nuevas tecnologías de la información y las comunicaciones (TIC) en nuestra sociedad es imparable: vivimos en una sociedad que dispone cada vez mas de mayor cantidad de documentos y aplicaciones electrónicas, de servicios de mensajería instantánea y de correo electrónico, de registros y portales de información telemáticos, de sistemas para la firma electrónica, de plataformas virtuales, etc. Se trata de un avance en el que nos encontramos inmersos y que está en constante

* Universidad Complutense de Madrid. 
cambio y actualización ${ }^{1}$. La Administración de Justicia no es ajena a este avance tecnológico y el legislador ha impulsado en los últimos años su modernización a través del uso de las nuevas tecnologías para conseguir que sea un servicio público de calidad, ágil, eficiente ${ }^{2}$ y cumplir con las exigencias del siglo XXI. En el marco europeo se han realizado importantes impulsos en este sentido como los siguientes:

- En el ámbito de la Unión Europea: la Comunicación de la Comisión Europea al Consejo al Parlamento y al Comité Económico y Social Europeo para la creación de la estrategia europea en materia de e-Justicia para lo cual la Comisión crea un portal con las siguientes funciones: "permitir el acceso a la información sobre los sistemas y los procedimientos judiciales e información práctica sobre las autoridades competentes y los medios para obtener asistencias judicial, remitir a los usuarios a las páginas de Internet de las instituciones, redes y registros judiciales europeos y proporcionar acceso directo a los procedimientos europeos elegidos" 3 .

- En el ámbito del Consejo de Europa: la Comisión Europea para la Eficacia de la Justicia ${ }^{4}$ (CEPEJ) que elabora evaluaciones de los sistemas judiciales cada dos años y determina las dificultades y propone planes concretos de mejora.

El proceso de modernización de la justicia española requiere la implantación y el desarrollo de las nuevas tecnologías en los juzgados y tribuna-

${ }^{1}$ El proceso de avance tecnológico no es reciente si consideramos los acontecimientos de los últimos doscientos años: la irrupción del teléfono a finales del siglo XIX, la televisión a mediados del siglo XX y la consolidación del uso de Internet y las telecomunicaciones a comienzos del siglo en el que nos encontramos.

${ }^{2}$ Según publica la propia web del Ministerio de Justicia a la que luego nos referiremos, el Plan 2015 para la aceleración de la Justicia en entornos digitales es la herramienta con la que se quiere conseguir una Justicia de calidad, ágil y eficiente al alcance de todos a lo largo del ejercicio 2015-2016. El objetivo principal que se persigue es conseguir que los ciudadanos dispongan de los medios que les permitan acceder a la Justicia a cualquier hora para informarse y realizar determinados trámites.

${ }^{3}$ Comunicación de la Comisión Europea al Consejo y el Parlamento y al Comité Económico y Social Europeo para la creación de la estrategia europea en materia de e-Justicia de 30 de mayo de 2008 publicada en el diario oficial COM (2008) 329.

${ }^{4}$ Creada por Resolución RES 12 del Comité de Ministros del Consejo de Europa en 2002. 
les al igual que la dotación de mayores medios personales y materiales, pero estos aspectos por sí solos no pueden resolver todos los problemas que aquejan a la Justicia espańola. Se requiere también la promulgación de un conjunto de medidas normativas, estructurales y orgánicas y procesales, bien encaminadas, profundas y armonizadas entre sí, para lograr un servicio público de calidad que garantice una tutela judicial efectiva, un servicio público accesible, transparente y eficiente que cuente con una organización adecuada con medios personales y materiales precisos para llevar a cabo su importante función.

En la actualidad estamos viviendo este proceso de cambio, se trata de un momento histórico y muy importante ya que se están llevando a cabo distintas iniciativas para la efectiva implantación de los medios electrónicos en la tramitación de los procedimientos judiciales que precisamente van a ser el objeto de breve referencia y comentario en este breve trabajo.

\section{BASES PARA LA MODERNIZACIÓN DE LA ADMINISTRACIÓN DE JUSTICIA EN ESPAÑA}

Hay que destacar que en cuarenta años años de democracia española el impulso modernizador de los diferentes gobiernos ha supuesto la mejora de algunos servicios públicos, pero no ha existido una acción concertada de todos los sectores implicados y todas las instituciones públicas orientada a mejorar la Justicia tal y como la que existe en la actualidad. Si analizamos las bases y la paulatina evolución en todos estos años encontramos importantes impulsos modernizadores que comentamos de forma breve a continuación.

La base competencial más importante a considerar en este proceso la encontramos en la Constitución Espańola que confiere competencias en materia de Justicia tanto al Gobierno como al Consejo General del Poder Judicial y a las Comunidades Autónomas, por tanto todos los interlocutores están obligados a colaborar para el éxito de la modernización.

A partir de los años 90 del siglo pasado aparecen los primeros impulsos modernizadores más contundentes. Podemos citar como un hito impor- 
tante la proclamación del Libro Blanco de la Justicia ${ }^{5}$ de 1997 que contenía referencias a "la aplicación al proceso de las nuevas tecnologías y las nuevas formas de documentación” y destacaba que "el perfeccionamiento progresivo de los sistemas de comunicación informática y electrónica ha de comportar las imprescindibles reformas de los procedimientos tradicionales de notificación y recepción de documentos con la consiguiente reducción de dilaciones". En el año 2002 la Carta de Derechos de los Ciudadanos ante la Justicia ${ }^{6}$ recogía una sección titulada "una justicia ágil y tecnológicamente avanzada” y proponía el impulso por parte de los poderes públicos del empleo y aplicación de medios tecnológicos avanzados en la actividad de la Administración de Justicia. Se apuntaba así necesariamente al objetivo deseado de la modernización si bien no se realizaron las correspondientes dotaciones presupuestarias ni se organizaron oportunamente las reformas legales y los medios personales y materiales en su consecución.

Desde el Consejo General del Poder Judicial se pusieron en marcha distintas iniciativas como la aprobación, el 12 de noviembre de 2008, del Plan de Modernización de la Administración de Justicia con las diversas acciones que se intentaron llevar a cabo:

1.- Implantación de la nueva oficina judicial

2.- Reestructuración de la planta y demarcación judicial

3.- Aplicación de las nuevas tecnologías

4.- Carta de Derechos y Plan de Transparencia Judicial

5.- Seguimiento y mejora de la actividad de los órganos judiciales

6.- Impulso de las reformas orgánicas procesales

7.- Estatuto de los miembros de la carrera judicial

8.- Organización territorial del Estado

En abril de 2009 el Pleno del Congreso de los Diputados aprobó una proposición que declaraba urgente impulsar un acuerdo social en materia de Justicia que englobara al conjunto de los grupos parlamentarios y facilitara el consenso con el gobierno y todos los agentes jurídicos y que se 1997.

${ }^{5}$ Libro Blanco de la Justicia aprobado por el Consejo General del Poder Judicial en

${ }^{6}$ Carta de Derechos de los Ciudadanos ante la Justicia aprobada por el Congreso de los Diputados en 2002. 
materializara en un calendario con un programa de actuación y una inversión concreta. El Consejo General del Poder Judicial se unió a este consenso al igual que las Comunidades Autónomas. A partir de ese momento se impulsaron desde el Gobierno los procesos legislativos necesarios para la reforma de la Ley Orgánica del Poder Judicial y las reformas procesales para la puesta en funcionamiento de la nueva oficina judicial y se inició la elaboración de un Plan integral que permitiese la modernización esperada.

El Gobierno aprobó en septiembre de 2009 el Plan Estratégico 20092012 de Modernización de la Justicia basado en tres políticas fundamentales ${ }^{7}$ : política de modernización, política de protección y transparencia y política de cooperación. Sus cuatro retos fundamentales eran: conseguir la interoperabilidad entre los sistemas tecnológicos, unificar esfuerzos por parte de las distintas administraciones e instituciones judiciales, cambiar la organización de los recursos personales y tramitar las reformas legales necesarias para conseguir modernizar la Administración de Justicia. Para ello se establecieron seis ejes estratégicos de actuación:

- Eje 1: Un servicio público de calidad.

- Eje 2: Una Justicia profesional y altamente cualificada.

- Eje 3: Una Justicia tecnológicamente avanzada.

${ }^{7}$ El Plan Estratégico, muy detallado, técnico y riguroso, pretendía der desarrollado en mayor detalle en planes anuales de actuación para programar eficazmente las distintas actuaciones. Se contempló una importante inversión para el año 2010 ascendente a 214 millones de euros aunque se prevé alcanzar los 600 millones en los años siguientes. En el período 2009-2012 se pusieron en marcha una serie de mecanismos, previstos coordinadamente en el Plan, para que la Administración de Justicia diera un cambio cualitativo sustancial y cumpla realmente con lo que se espera de ella, de forma que el sistema judicial cambie su inercia y se instale definitivamente en el siglo XXI. El Plan Estratégico de Modernización del Sistema de Justicia agrupó tanto reformas normativas como cambios organizativos y mejoras tecnológicas. El objetivo concreto es dar una respuesta a la demanda de la sociedad española al completo de disponer de un servicio público de Justicia ágil, transparente, responsable y conforme con los valores establecidos constitucionalmente. En la medida en que la consecución de este objetivo requiere una adecuada sistematización y control de los cambios, el Plan incluye un instrumento de seguimiento y evaluación específico al que denomina Plan Operativo Anual (comúnmente conocido como POA). En el mismo se recogían una serie de actividades que se llevaron a cabo, cada año, para ejecutar el Plan de Modernización y fue una clara manifestación del compromiso firme por parte del Ministerio de Justicia para afrontar la actualización del sistema judicial de acuerdo a unas premisas realistas y ejecutables a medio plazo. 
- Eje 4: Un servicio público orientado a los ciudadanos.

- Eje 5: Una política basada en la colegiación de esfuerzos.

- Eje 6: Un fortalecimiento la cooperación jurídica internacional.

En aquel momento para conseguir una Justicia tecnológicamente avanzada se propone hacer lo posible que los juzgados y tribunales puedan trabajar en red, la creación y desarrollo del "Esquema Judicial de Interoperabilidad y Seguridad" para permitir el funcionamiento integrado y conjunto de las distintas aplicaciones informáticas al servicio de la Administración de Justicia y de este modo la interoperabilidad entre órganos judiciales, lograr el objetivo "papel 0", desplegar al completo el sistema seguro de intercambio de documentos (Lexnet) que permite que todas las comunicaciones procesales se realicen por vía electrónica, la creación del Expediente Judicial Electrónico mediante la implantación de un nuevo Sistema de Gestión Procesal, el desarrollo de una Plataforma de Firma Electrónica propia de la Administración de Justicia e incrementar los medios de grabación audiovisual y videoconferencia en las vistas y mejora de la gestión del almacenamiento de las piezas de convicción a través del sistema de identificación por radiofrecuencia.

Además, para conseguir un servicio público de Justicia moderno y eficaz orientado a las personas, se pretendía fortalecer la protección de los derechos ciudadanos, promover la transparencia de la Administración de Justicia mediante el fortalecimiento del sistema de estadística judicial y publicar, por parte del Ministerio de Justicia, toda aquella información sobre su actuación relevante para el derecho de acceso a la información pública de los ciudadanos.

Posteriormente se implementó el Plan 2012-2015 de la Secretaría General de la Administración de Justicia ${ }^{8}$ que describió una batería

${ }^{8}$ Con el objetivo de facilitar su difusión, esta actualización del Plan de Acción se presenta en formato papel y en formato digital, disponible para su descarga desde el Portal del Ministerio http://www.mjusticia.gob.es (2016-08-22) y en el Portal de la Administración de Justicia https://www.administraciondejusticia.gob.es/ (2016-08-22). Las actuaciones y proyectos que desarrolla están en línea con las directrices de la Secretaría General de la administración de Justicia: 1. Introducir una amplia reorganización de la actividad judicial que permita, a través de la Oficina Judicial y la implantación de los tribunales de instancia, una mejor asignación de recursos y una pronta resolución de los litigios; 2. Desarrollo de aplicaciones y servicios que faciliten la gestión procesal de los asuntos sometidos a los Tri- 
importante de reformas para lograr un servicio público de calidad que supuso la reforma de la Ley Orgánica del Poder Judicial, la Ley de Demarcación y Planta, la Ley de la Jurisdicción Voluntaria, el Estatuto de la Víctima, la Ley de Enjuiciamiento Criminal y el Código Penal, entre otras leyes. El citado Plan se estructura alrededor de Programas que engloban las principales actuaciones y proyectos que marcarán la actividad de la Secretaría General de la Administración de Justicia para los próximos años y que agrupa de la siguiente forma en cuatro Programas:

- Programa 1: Organización de la actividad judicial.

- Programa 2: Infraestructura tecnológica y servicios de la actividad judicial.

- Programa 3: Cooperación entre servicios de la actividad judicial.

- Programa 4: Procedimientos de gestión de la Administración de Justicia.

Fruto de este Plan podemos destacar que actualmente se mantiene dentro de la web del Ministerio de Justicia un apartado específico para la "modernización de la Justicia" en la que se abordan varios ejes diferentes a los que se dedica atención preferente:

- El Expediente Judicial Electrónico, su implantación supone la definitiva superación de una justicia basada en el papel, un importante ahorro en recursos y una simplificación de la gestión, lo que repercute en una mayor celeridad en la adopción de las resoluciones y, en consecuencia, en una reducción de los tiempos de espera. Con el Expediente Judicial Electrónico se avanza, de forma decidida, en la senda marcada por la Ley 18/2011, de 5 de julio, reguladora del uso de las tecnologías de la información y la comunicación en la Administración de Justicia, cuyo Título IV fija las

\footnotetext{
bunales de Justicia, así como la progresiva implantación de nuevas metodologías de trabajo; 3. Fomentar la cooperación entre las Administraciones competentes, en permanente colaboración con el Consejo General del Poder Judicial, Fiscalía General del Estado y Comunidades Autónomas, así como la Cooperación Internacional; 4. Racionalizar la creación y funcionamiento de los Tribunales de Justicia, a través de una profunda remodelación de la demarcación y planta judicial y de la monitorización de la estadística judicial; 5. Potenciar la actuación del Cuerpo Superior Jurídico de Secretarios Judiciales, así como de los demás cuerpos al servicio de la Administración de Justicia, aplicando una gestión eficaz de los recursos humanos.
} 
condiciones para hacer posible la íntegra tramitación electrónica de los procedimientos judiciales y dedica el Capítulo II a definir y regular el Expediente Judicial Electrónico.

- La modernización tecnológica de la Justicia a través de LexNet, una plataforma de intercambio seguro de información entre los órganos judiciales y una gran diversidad de operadores jurídicos que, en su trabajo diario, necesitan intercambiar documentos judiciales (notificaciones, escritos y demandas). Han transcurrido varios años desde su implantación y LexNet se ha convertido en un instrumento de trabajo seguro tan habitual en el ámbito de las comunicaciones judiciales, como el teléfono, el fax o el correo electrónico. El sistema, que empezó a funcionar en el año 2004, cuenta en la actualidad con más de 52.000 usuarios, es utilizado en más de 3.500 órganos judiciales y ha permitido intercambiar más de 160.000.000 de comunicaciones.

Lexnet, tal y como su nombre indica (la Ley en la Red), pretende extender gran parte de los servicios que ofrece la Administración de Justicia a través de Internet, haciendo que éstos sean accesibles desde cualquier parte y en cualquier momento. En la actualidad el sistema permite:

- El intercambio seguro de información

- El uso de firma electrónica reconocida

- Acceso vía web

- Funcionamiento 24 horas 7 días a la semana

- Ahorro de papel

- Inmediatez en las comunicaciones

- La modernización del lenguaje jurídico, en este sentido la Carta de Derechos del Ciudadano ante la Justicia9 ya incidía en este aspecto por lo que se constituyó la Comisión para la Modernización del lenguaje jurídico que, en septiembre de 2011, presentó su informe al Consejo de Ministros ${ }^{10}$ y que continúa operativa.

${ }^{9}$ Que fue aprobada por unanimidad en el Congreso de los Diputados el 16 de abril de 2002.

${ }^{10} \mathrm{El}$ informe contiene recomendaciones sobre corrección lingüística y ofrece una guía de ejemplos para mejorar la redacción de los escritos jurídicos. En él se reconoce que los 
- Y el Plan de acción para la aceleración de la Justicia en entornos digitales, presentado el 17 marzo de 2015 por el Ministerio de Justicia con los siguientes objetivos estratégicos:

- Maximizar la eficacia y eficiencia de la Justicia como servicio público

- Orientar el servicio al ciudadano y sus nuevas demandas

- Asentar la transformación en una cultura de innovación y gestión

Para conseguirlo plantea tres ejes de transformación de la justicia: transformación de la justicia a través de un cambio en el funcionamiento (justicia digital), la interacción con los ciudadanos (justicia abierta, y la gestión y cultura interna (justicia innovadora). Sus bases resultan claras: modernizar, agilizar e informatizar la justicia. Con este Plan estamos asistiendo a un fenómeno histórico: el que supone la modernización definitiva de la Administración de Justicia con los procesos de informatización y digitalización.

El plan 2015 de aceleración de la Justicia en entornos digitales disponible en la web del Ministerio de Justicia ${ }^{11}$ es la herramienta con la que se

ciudadanos consideran críptico el lenguaje judicial y propone sustituir los particularismos lingüísticos por términos del lenguaje común, siempre que sea posible. Las recomendaciones propuestas por la Comisión van más allá de la pura ortografía y pretenden crear un marco institucional para devolver la relevancia que el uso del lenguaje nunca debió de perder. El informe se estructura en dos tipos principales de recomendaciones: a los profesionales: recomendaciones básicas sobre la base de los errores gramaticales y de sintaxis más comunes; a las instituciones a quienes corresponde impulsar medidas que permitan acercar el lenguaje jurídico al ciudadano y poner al alcance de nuestros profesionales del derecho medios suficientes para la búsqueda de la excelencia en su expresión lingüística.

${ }^{11}$ Puede verse la presentación en este link:

http://www.mjusticia.gob.es/cs/Satellite/Portal/1292427354807?blobheader=application\%2Fpdf\&blobheadername1=Contentdisposition\&blobheadervalue $1=$ attachment $\% 3 B+f i l e n a m e \% 3 D$ (2016-08-22). A partir de la segunda mitad de 2015, tal y como recoge el Plan, los nacimientos y las defunciones dejan de notificarse en persona en el Registro Civil y será personal sanitario quien lo haga de forma telemática. Esto permitirá ahorrar más de 64 millones de euros al año, al eliminarse 850.000 trámites presenciales. Tras el verano de 2015 se dispone de una primera versión de la aplicación para móviles de LEXNET, la plataforma de intercambio seguro de in-formación entre órganos judiciales y operadores jurídicos que el año pasado permitió ahorrar 94 millones de euros gracias a los 46 millones de notificaciones que se realizaron a través de ella. En otońo se pondrá en marcha la nueva 
quiere conseguir modernizar la Administración de Justicia para acercarla al ciudadano y se concreta en dos ideas: justicia sin papel y justicia en $\operatorname{red}^{12}$.

\section{REFORMAS PROCESALES CIVILES RELACIONADAS \\ CON LA INFORMATIZACIÓN DE LA ADMINISTRACIÓN DE JUSTICIA}

Centrándonos ya en los aspectos procesales para la modernización de la administración de justicia con las nuevas tecnologías se han operado recientemente importantes reformas dentro de las iniciativas de la e-Justicia a las que nos referíamos al comienzo de este trabajo, como la introducción de la firma electrónica, la grabación de las vistas que ya se encontraba prevista desde 2000 en el ámbito civil, la generalización del uso de la videoconferencia en los procedimientos, la mediación on line, la creación de plataformas virtuales para comunicar a los distintos operadores jurídicos.

Pero para realizar la implementación de las reformas hay que considerar también que la justicia es un servicio público y que, por tanto, los programas de mejora y modernización que se aborden deben implementarse salvaguardando el funcionamiento diario normal, lo que obliga a ir

sede judicial electrónica, a través de la que cualquier persona inmersa en un proceso judicial podrá tener conocimiento de cómo van sus trámites con la Justicia 24 horas al día durante los 7 días de la semana. A mediados de año la comunicación de requisitorias a las Fuerzas y Cuerpos de Seguridad del Estado se hará mediante correo electrónico, tal y como se realiza ya en 72 juzgados de Madrid. Durante 2015 continua la implantación del expediente digital en la Audiencia Nacional y se extenderá al resto del territorio Ministerio comenzando en los juzgados de Cuenca a mediados de año. A principios de 2016 será obligatoria la comunicación electrónica entre los colectivos jurídicos y las sedes judiciales. En palabras del Ministro de Justicia, Rafael Catalá, "Esta es la Justicia en la que el Ministerio está trabajando" en la que se priman "la tecnología y la innovación como medios para transformar este servicio público y colocarlo a la altura de lo que demandan los ciudadanos". Asistimos pues a un proceso histórico para nuestra Administración de Justicia.

${ }^{12}$ Puede accederse al vídeo divulgativo publicado con este motivo en el siguiente enlace en el que el Ministro de Justicia, Sr. Catalá, presenta el programa y las acciones que se están implementando para ponerlo en marcha: https://www.administraciondejusticia. gob.es/paj (2016-08-22). 
desarrollando poco a poco reformas graduales que supongan mejoras paulatinas mientras se mantiene el servicio.

Ha sido con las estas últimas reformas cuando ya se está logrando la aplicación generalizada de los medios electrónicos en la tramitación de los procedimientos y se ha acrecentado su protagonismo procedimental. Citaremos seguidamente los más destacados cambios legales ${ }^{13}$ centrándonos en el ámbito civil, que es el que nos interesa más concretamente a los efectos del presente trabajo.

En orden a conseguir la efectiva informatización del proceso civil se han requerido sucesivas y profundas reformas que han ido introduciendo poco a poco cambios sustanciales en el articulado de la Ley de Enjuiciamiento Civil, que recordemos resulta subsidiaria respecto del resto del ordenamiento procesal en los distintos órdenes jurisdiccionales.

Ya la Ley de Enjuiciamiento Civil en su redacción original ya reconoce el uso de los medios electrónicos en el año 2000 y en su Exposición de Motivos se indicaba que se pretende "abrir la puerta a la presentación de escritos y documentos y a los actos de notificación por medios electrónicos, telemáticos y otros semejantes", si bien ya desde 1994 el art. 230 de la Ley Orgánica del Poder Judicial establecía que "los Juzgados y Tribunales podrán utilizar cualesquiera medios técnicos, electrónicos, informáticos y telemáticos, para el desarrollo de su actividad y ejercicio de sus funciones, con las limitaciones que a la utilización de tales medios establece la Ley Orgánica 5/1992, de 29 de octubre y demás leyes que resulten de aplicación. Las personas que demanden la tutela judicial de sus derechos e interese podrán relacionarse con la Administración de Justicia a través de los medios técnicos a que se refiere el apartado primero cuando sean com-

${ }^{13}$ Por lo que respecta al ámbito penal la Ley Orgánica 13/2015, de 5 de octubre, de modificación de la Ley de Enjuiciamiento Criminal incorpora nuevas medidas de investigación tecnológica como consecuencia de la proliferación del número de delitos relacionados con el uso de las nuevas tecnologías. En el ámbito administrativo podemos destacar como hito importante la publicación de la Ley 39/2015, de 1 de octubre, del Procedimiento Administrativo Común de las Administraciones Públicas, que pretende implantar una Administración totalmente electrónica con registros telemáticos y un punto de acceso general electrónico de la Administración. En el ámbito laboral la reforma de la Ley de Procedimiento Laboral también ha supuesto un impulso en lo que a utilización de tecnologías de la información se refiere. 
patibles con los que dispongan los Juzgados y Tribunales y se respeten las garantías y requisitos previstos en el procedimiento de que se trate".

Podemos citar otros hitos legales importantes sobre la incorporación de las nuevas tecnologías en el proceso civil llevadas a cabo paulatinamente como la Ley 34/2002 de servicios de la Sociedad de la Información y de la contratación por vía electrónica; la Ley 59/2003 que regula la eficacia jurídica de la firma electrónica y la prestación de servicios de certificación; la Instrucción 2/2003 del Pleno del Consejo General del Poder Judicial por la que se aprueba el Código de Conducta para usuarios de equipos y sistemas informáticos al servicio de la Administración de Justicia; también la Ley 19/2006 mediante la que se amplían los medios de tutela de los derechos de propiedad intelectual e industrial estableciendo normas procesales para facilitar la aplicación de diversos reglamento comunitarios y cumplir las exigencias de la Directiva 2004/48/CE; la reforma de la Ley 41/2007 de la regulación del mercado hipotecario y otras normas del sistema hipotecario y financiero, de regulación de las hipotecas inversas y el seguro de dependencia y por la que se establece determinada norma tributaria que introduce aplicaciones telemáticas para la aportación de documentos públicos y privados en soporte electrónico; Real Decreto 84/2007, de 26 de enero, sobre implantación en la Administración de Justicia del sistema informático de telecomunicaciones LexNET para la presentación de escritos y documentos, el traslado de copias y la realización de actos de comunicación procesal por medios telemáticos; la Ley 11/2007, de 22 de junio, de acceso electrónico de los ciudadanos a los Servicios Públicos, estas Administraciones han sido pioneras en el uso de las nuevas tecnologías en el ámbito de las Administraciones Públicas y se han definido en los últimos años diversos sistemas de notificaciones impulsados por varios órganos de la Administración General del Estado; y la Ley 25/2007 de conservación de datos relativos a las comunicaciones electrónicas y a las redes públicas de comunicaciones, la Ley 13/2009 de reforma de la legislación procesal para la implantación de la nueva Oficina Judicial.

Ya en esta década la Ley 4/2011, de 24 de marzo, de modificación de la Ley de Enjuiciamiento Civil incorpora previsiones para la utilización de nuevas tecnologías durante la tramitación de un proceso monitorio europeo o de escasa cuantía. 
Resulta singularmente relevante también la Ley 18/2011 de 5 de julio reguladora del uso de las tecnologías de la información y la comunicación en la Administración de Justicia, que pretende conseguir la eficacia de la justicia y regula el deber de utilizar los medios electrónicos para los profesionales de la justicia y de las oficinas judiciales, así como la obligación de las Administraciones competentes de dotar de estos medios y el derecho de los ciudadanos a relacionarse electrónicamente con la Administración de Justicia.

Además la Ley 5/2012, de 6 de julio, de mediación en asuntos civiles y mercantiles recoge especialmente un proceso de mediación simplificado por medios electrónicos cuando se trate de reclamaciones de cantidad que no excedan de 600 euros (son los denominador ODR on line dispute resolution).

Conviene destacar también el Real Decreto 892/2013, de 15 de noviembre, por el que se regula el Registro Público concursal que depende del Ministerio de Justicia para dar publicidad de resoluciones y asientos relacionados con el proceso concursal.

La Ley Orgánica 7/2015 de 21 de julio de reforma de la LOPJ obliga a utilizar medios técnicos y electrónicos a los Juzgados, Tribunales y Fiscalías ${ }^{14}$.

También la Ley 19/2015, de 13 de julio, de medidas de reforma administrativa en el ámbito de la Administración de Justicia y el Registro Civil ha supuesto la aparición del Portal de subastas electrónicas ${ }^{15}$.

Más recientemente la Ley 42/2015, de 5 de octubre, de Reforma de la Ley de Enjuiciamiento Civil establece en su Preámbulo que "constituye una necesidad imperiosa acometer una reforma en profundidad de las diferentes actuaciones procesales para generalizar y dar mayor relevancia al uso

${ }^{14}$ Ley Orgánica 7/2015, de 21 de julio, por la que se modifica la L.O. 6/1985, de 1 de julio, del Poder Judicial, que dio nueva redacción a su artículo 230, que establece que "Los Juzgados y Tribunales y las Fiscalías están obligados a utilizar cualesquiera medios técnicos, electrónicos, informáticos y telemáticos, puestos a su disposición para el desarrollo de su actividad y ejercicio de sus funciones...."

${ }^{15}$ A nivel europeo para promover estos cambios puede citarse el Reglamento (UE) 910/2014 del Parlamento Europeo y del Consejo, de 23 de julio de 2014, relativo a la identificación electrónica y los servicios de confianza para las transacciones electrónicas en el mercado interior y por el que se deroga la Directiva 1999/93/CE. 
de los medios telemáticos o electrónicos, otorgando carácter subsidiario al soporte papel", anunciando que a partir de julio de 2016 los profesionales de la justicia, los órganos judiciales y las fiscalías están obligados a utilizar sistemas telemáticos o electrónicos para la presentación de escritos y documentos y la realización de actos de comunicación procesal y, además, que a partir de esa fecha las administraciones con competencias en materia de justicia estarán obligadas a realizar la tramitación electrónica de los procedimientos judiciales conforme ya había referido la Ley 17/2011 reguladora del uso de las tecnologías de la comunicación y la información en la Administración de Justicia ${ }^{16}$.

Quizá una de las reformas de mayor relevancia ha sido el Real Decreto 1065/2015, de 27 de noviembre, sobre comunicaciones electrónicas en la Administración de Justicia en el ámbito territorial del Ministerio de Justicia y por el cual se regula el sistema Lexnet ${ }^{17}$

Resulta también relevante en este sentido el contenido del Real Decreto $1011 / 2015$ de 6 de noviembre que establece un sistema único de constitución telemática de los depósitos necesarios para participar en todo tipo de subastas, notariales y judiciales, en sede electrónica.

Seguidamente destacamos las más recientes e importantes innovaciones legislativas en el ámbito del proceso civil que se están implantando en la actualidad.

${ }^{16}$ Ley 42/2015, de 5 de octubre, de reforma de la Ley de Enjuiciamiento Civil, cuya Disposición Adicional Primera prevé que a partir del 1 de enero de 2016 es obligatorio para todos los profesionales de la justicia y órganos y oficinas judiciales y fiscales el uso de los sistemas telemáticos existentes en la Administración de Justicia para la presentación de escritos y documentos y la realización de actos de comunicación procesal en los términos de la ley procesal y de la Ley 18/2011, respecto de los procedimientos que se inicien a partir de esta fecha.

${ }^{17}$ Este Real Decreto sustituye al anterior 84/2007, de 26 de enero, sobre implantación en la Administración de Justicia del sistema informático de telecomunicación Lexnet para la presentación de escritos y documentos, el traslado de copias y la realización de actos de comunicación procesal por medios telemáticos. 


\subsection{OBLIGACIÓN DE PRESENTACIÓN TELEMÁTICA O ELECTRÓNICA DE ESCRITOS Y DOCUMENTOS Y ENVÍO Y RECEPCIÓN DE ACTOS DE COMUNICACIÓN}

Según el art. 273.1 de la Ley de Enjuiciamiento Civil sobre la presentación de escritos y documentos "Todos los profesionales de la justicia están obligados al empleo de los sistemas telemáticos o electrónicos existentes en la Administración de Justicia para la presentación de escritos, iniciadores o no, y demás documentos, de forma tal que esté garantizada la autenticidad de la presentación y quede constancia fehaciente de la remisión y la recepción íntegras, así como de la fecha en que éstas se hicieren”. Igualmente se establece en el párrafo segundo del citado artículo que "Las personas que no estén representadas por procurador podrán elegir en todo momento si actúan ante la Administración de Justicia a través de medios electrónicos o no, salvo que estén obligadas a relacionarse a través de medios electrónicos con la misma. El medio elegido podrá ser modificado en cualquier momento".

En cuanto a los sujetos obligados ${ }^{18}$ a presentar los documentos y escritos por esta vía el mismo precepto establece que "En todo caso, estarán obligados a intervenir a través de medios electrónicos con la Administración de Justicia, al menos, los siguientes sujetos: a) Las personas jurídicas; b) Las entidades sin personalidad jurídica; c) Quienes ejerzan una actividad profesional para la que se requiera colegiación obligatoria para los trámites y actuaciones que realicen con la Administración de Justicia en ejercicio de dicha actividad profesional; d) Los notarios y registradores; e) Quienes representen a un interesado que esté obligado a relacionarse electrónicamente con la Administración de Justicia; f) Los funcionarios de las Administraciones Públicas para los trámites y actuaciones que realicen por razón de su cargo".

${ }^{18}$ Se establece legalmente que el incumplimiento del deber del uso de las tecnologías previsto en este artículo o de las especificaciones técnicas que se establezcan conllevará que el letrado de la Administración de Justicia conceda un plazo máximo de cinco días para su subsanación y también que si no se subsana en este plazo, los escritos y documentos se tendrán por no presentados a todos los efectos. 


\subsection{CONTENIDO DE LOS ESCRITOS Y DOCUMENTOS PRESENTADOS POR VÍA TELEMÁTICA O ELECTRÓNICA}

El apartado cuarto del citado artículo 273 de la Ley de Enjuiciamiento Civil establece que los escritos y documentos presentados por vía telemática o electrónica indicarán:

- El tipo y número de expediente y año al que se refieren

- Irán debidamente foliados mediante un índice electrónico que permita su debida localización y consulta. L

- La presentación se realizará empleando firma electrónica reconocida y se adaptará a lo establecido en la Ley 18/2011, de 5 de julio, reguladora del uso de las tecnologías de la información y la comunicación en la Administración de Justicia.

- Únicamente de los escritos y documentos que se presenten vía telemática o electrónica que den lugar al primer emplazamiento, citación o requerimiento del demandado o ejecutado, se deberá aportar en soporte papel, en los tres días siguientes, tantas copias literales cuantas sean las otras partes.

Paralelamente la Ley Orgánica del Poder Judicial reformada por la Ley Orgánica 7/2015 obliga a los Juzgados y Tribunales y las Fiscalías a la utilización de medios técnicos, electrónicos, informáticos y telemáticos para el desarrollo de sus funciones y previene que el uso de estos sistemas debe hacerse respectando en todo caso las disposiciones de la Ley Orgánica de Protección de Datos de Carácter Personal y otras leyes que resulten de aplicación. En cuanto a la validez de estos escritos y su eficacia de los documentos presentados por estos medios el art. 230.2 de la LOPJ los equipara con los documentos originales siempre y cuando quede garantizada su autenticidad e integridad.

Sin perjuicio de todo lo anterior se mantiene la obligación de presentar en soporte papel los escritos y documentos cuando expresamente lo indique la ley y acompańando siempre tantas copias literales cuantas sean las otras partes. 


\subsection{FORMA DE PRESENTACIÓN DE ESCRITOS POR SISTEMAS TELEMÁTICOS O ELECTRÓNICOS}

El art. 135 de la LEC establece en cuanto a la presentación de escritos y los requisitos de tiempo que cuando las oficinas judiciales y los sujetos intervinientes en un proceso ${ }^{19}$ estén obligados al empleo de los sistemas telemáticos o electrónicos existentes en la Administración de Justicia conforme al artículo 273 , remitirán y recibirán todos los escritos, iniciadores o no, y demás documentos a través de estos sistemas, salvo las excepciones establecidas en la ley, de forma tal que se reúnan los siguientes requisitos:

- Quede garantizada la autenticidad de la comunicación

- Quede constancia fehaciente de la remisión y su fecha

- Y quede constancia también de la recepción íntegra y su fecha

\subsection{FECHA Y HORA DE PRESENTACIÓN DE LOS ESCRITOS Y DOCUMENTOS APORTADOS POR SISTEMAS ELECTRÓNICOS Y TELEMÁTICOS Y SU JUSTIFICACIÓN}

Establece la Ley de Enjuiciamiento Civil que se podrán presentar escritos y documentos en formato electrónico todos los días del año durante las veinticuatro horas. Es ésta una de las principales ventajas de la nueva regulación que deja al margen los horarios oficiales de atención en los tribunales de justicia para este menester.

En cuanto a la emisión de recibo de la presentación se establece que presentados los escritos y documentos por medios telemáticos, se emitirá automáticamente recibo por el mismo medio, con expresión del número de entrada de registro y de la fecha y la hora de presentación, en la que se tendrán por presentados a todos los efectos. En caso de que la presentación tenga lugar en día u hora inhábil a efectos procesales conforme a la ley, se entenderá efectuada el primer día y hora hábil siguiente.

${ }^{19}$ Según el propio artículo 135 de la LEC ésto será también de aplicación a aquellos intervinientes que, sin estar obligados, opten por el uso de los sistemas telemáticos o electrónicos. 


\subsection{INTERRUPCIONES DEL SERVICIO DE COMUNICACIONES TELEMÁTICAS O ELECTRÓNICAS}

Cuando la presentación de escritos perentorios dentro de plazo por los medios telemáticos o electrónicos a que se refiere el apartado anterior no sea posible por interrupción no planificada del servicio de comunicaciones telemáticas o electrónicas, siempre que sea posible se dispondrán las medidas para que el usuario resulte informado de esta circunstancia, así como de los efectos de la suspensión, con indicación expresa, en su caso, de la prórroga de los plazos de inminente vencimiento. El remitente podrá proceder, en este caso, a su presentación en la oficina judicial el primer día hábil siguiente acompañando el justificante de dicha interrupción.

En los casos de interrupción planificada deberá anunciarse con la antelación suficiente, informando de los medios alternativos de presentación que en tal caso procedan.

Si el servicio de comunicaciones telemáticas o electrónicas resultase insuficiente para la presentación de los escritos o documentos, se deberá presentar en soporte electrónico en la oficina judicial ese día o el día siguiente hábil, junto con el justificante expedido por el servidor de haber intentado la presentación sin éxito. En estos casos, se entregará recibo de su recepción.

La presentación de escritos y documentos, cualquiera que fuera la forma, si estuviere sujeta a plazo, podrá efectuarse hasta las quince horas del día hábil siguiente al del vencimiento del plazo.

En las actuaciones ante los tribunales civiles, no se admitirá la presentación de escritos en el juzgado que preste el servicio de guardia.

\subsection{TRASLADO DE LAS COPIAS A LAS OTRAS PARTES}

Se regula en los art. 274 y siguientes de la LEC según que esté o no representada la parte por procurador o no:

Cuando las partes no actúen representadas por procurador, firmarán las copias de los escritos y documentos que presenten, respondiendo de su exactitud, y dichas copias se entregarán por el 
secretario judicial a la parte o partes contrarias. Si la presentación se realizara por medios telemáticos por estar obligados o haber optado por ello, siempre que cumplan los presupuestos y requisitos exigidos, el traslado de las copias a las demás partes se realizará por la oficina judicial por el medio que proceda.

- Cuando las partes estuvieren representadas por procurador, cada uno de éstos deberá trasladar a los procuradores de las restantes partes las copias de los escritos y documentos que presente al tribunal. El traslado de copias de los escritos y documentos presentados de forma telemática, se hará por medios telemáticos de forma simultánea a la presentación y se entenderá efectuado en la fecha y hora que conste en el resguardo acreditativo de su presentación. En caso de que el traslado tenga lugar en día y hora inhábil a efectos procesales conforme a la ley se entenderá efectuado el primer día y hora hábil siguiente.

\subsection{APODERAMIENTO APUD ACTA}

En materia de representación también se puede realizar el apoderamiento apud acta por los nuevos medios electrónicos. En este sentido el art. 32 bis de la Ley reguladora de las tecnologías de la información y la comunicación establece que los apoderamientos inscritos en los archivos electrónicos tienen una validez de cinco años que se computan desde la fecha de inscripción. Para acreditar la representación procesal se aportará copia electrónica del poder notarias de representación conferido al procurador, copia electrónica de la representación otorgada por comparecencia apud acta ante el Letrado de la Administración de Justicia o el apoderamiento podrá acreditarse presentando la certificación de su inscripción en el archivo electrónico de apoderamientos apud acta.

\subsection{ENVÍO Y RECEPCIÓN DE ACTOS DE COMUNICACIÓN}

En este sentido el art. 162 de la LEC estable que: Cuando las oficinas judiciales y las partes o los destinatarios de los actos de comunicación estén 
obligados a enviarlos y recibirlos por medios electrónicos, telemáticos, infotelecomunicaciones o de otra clase semejante, que permitan el envío y la recepción de escritos y documentos, de forma tal que esté garantizada la autenticidad de la comunicación y de su contenido y quede constancia fehaciente de la remisión y recepción íntegras y del momento en que se hicieron, o cuando los destinatarios opten por estos medios, los actos de comunicación se efectuarán por aquellos, con el resguardo acreditativo de su recepción que proceda.

Los jueces podrán utilizar direcciones electrónicas habilitadas al efecto y otros datos como números de teléfono para localizar a las partes en el proceso si fuera necesario. En consonancia con ello en diciembre de 2015 el Ministerio de Justicia ha implantado un sistema de avisos por SMS para señalamientos de juicios y ya había otro servicio SMS de trámites procesales para víctimas de violencia de género que se puso en marcha como experiencia piloto en un Juzgado de Violencia de Género en Albacete y que ha contabilizado más de 200 avisos

Resulta importante destacar que los profesionales y destinatarios obligados a utilizar estos medios, así como los que opten por los mismos, deberán comunicar a las oficinas judiciales el hecho de disponer de los medios antes indicados y la dirección electrónica habilitada a tal efecto.

El precepto cita también un aspecto importante más y es que en el Ministerio de Justicia se constituirá un registro accesible electrónicamente de los medios indicados y las direcciones correspondientes a los organismos públicos y profesionales obligados a su utilización.

Resulta también destacable a efectos de notificaciones que, cuando conste la correcta remisión del acto de comunicación por dichos medios técnicos, si transcurren tres días sin que el destinatario acceda a su contenido, se entenderá que la comunicación ha sido efectuada legalmente desplegando plenamente sus efectos ${ }^{20}$. Y que no se practicarán actos de

${ }^{20}$ Se exceptuarán aquellos supuestos en los que el destinatario justifique la falta de acceso al sistema de notificaciones durante ese periodo. Si la falta de acceso se debiera a causas técnicas y éstas persistiesen en el momento de ponerlas en conocimiento, el acto de comunicación se practicará mediante entrega de copia de la resolución. En cualquier caso, la notificación se entenderá válidamente recibida en el momento en que conste la posibilidad de acceso al sistema. No obstante, caso de producirse el acceso transcurrido dicho plazo 
comunicación a los profesionales por vía electrónica durante los días del mes de agosto, salvo que sean hábiles para las actuaciones que corresponda.

En este sentido debemos llamar la atención sobre la necesidad de compatibilidad entre los medios informáticos que se empleen por los órganos jurisdiccionales y las aplicaciones electrónicas utilizadas por los ciudadanos que solicitan la tutela judicial de sus derechos e intereses, pues es necesario asegurar que los programas informáticos que se emplean puedan reconocer los distintos formatos, por ello Lexnet intenta, con sus potencialidades, solucionar este aspecto.

\subsection{LEXNET Y SU OBJETIVO "PAPEL CERO"}

El Ministerio de Justicia pretende conseguir centralizar en la plataforma Lexnet la información entre órganos judiciales y con órganos judicia$\operatorname{les}^{21}$. Se trata de una plataforma de intercambio seguro de información mediante técnicas criptográficas entre los órganos judiciales y una gran diversidad de operadores jurídicos ${ }^{22}$ garantizando la presentación de escritos y documentos y la recepción de actos de comunicación, sus fechas de emisión, recepción o acceso y la integridad de las mismas con identificación del remitente y destinatario de las mismas.

En cuanto a su valoración y funcionamiento se destaca que han transcurrido varios ańos desde su implantación y Lexnet se ha convertido en un instrumento de trabajo seguro tan habitual en el ámbito de las comunicaciones judiciales, como el teléfono, el fax o el correo electrónico. El sistema empezó a funcionar en el año 2004 y cuenta con una gran cantidad de

pero antes de efectuada la comunicación mediante entrega, se entenderá válidamente realizada la comunicación en la fecha que conste en el resguardo acreditativo de su recepción.

${ }^{21}$ Según se contiene en el articulado del Real Decreto 1065/2015, de 27 de noviembre por el que se regula Lexnet, entre otras cuestiones.

${ }^{22}$ En cuanto a los usuarios que actualmente están utilizando el sistema: los órganos judiciales y Oficinas de Registro y Reparto (Secretario Judiciales), Procuradores y Colegio de Procurado-res, Abogacía del Estado, Abogacías dependientes de la Aboga-cía del Estado (FOGASA, SEPES, AEAT y Consorcio Compensación de Seguros), Abogacía Comunidad, Abogacía SJSS, Fiscalías, Abogados y Colegios de Abogados, Graduados Sociales, Comisiones de Asistencia Jurídica Gratuita, etc. 
usuarios $^{23}$. Es utilizado en más de 3.500 órganos judiciales y ha permitido intercambiar más de 160.000.000 de comunicaciones.

El sistema Lexnet es un medio de transmisión seguro de información que permite el intercambio seguro de información, garantiza la autenticidad e integridad de la información, el uso de firma electrónica reconocida, garantiza la fecha y hora de envío y recepción de los escritos, justificantes de presentación de escritos con plena validez jurídica, prestar servicio estarán en funcionamiento durante las veinticuatro horas del día, todos los días del año.

El sistema Lexnet presta las siguientes funcionalidades según el art. 14 del Real Decreto 1065/2015, de 27 de noviembre:

- La presentación y transporte de escritos procesales y documentos que con los mismos se acompañen, así como su distribución y remisión al órgano u oficina judicial o fiscal encargada de su tramitación.

- La gestión del traslado de copias, de modo que quede acreditado en las copias la fecha y hora en que se ha realizado efectivamente el traslado a los restantes Procuradores personados y la identidad de éstos, de conformidad con lo previsto en las leyes procesales.

${ }^{23}$ Son usuarios del sistema: Ministerio Fiscal, Funcionarios del Cuerpo Superior Jurídico de Letrados de la Administración de Justicia, Funcionarios de los Cuerpos de Médicos Forenses, de Facultativos del Instituto Nacional de Toxicología y Ciencias Forenses, de Técnicos Especialistas del Instituto Nacional de Toxicología y Ciencias Forenses y de Ayudantes de Laboratorio del Instituto Nacional de Toxicología y Ciencias Forenses, Funcionarios del Cuerpo de Gestión Procesal y Administrativa, Funcionarios del Cuerpo de Tramitación Procesal y Administrativa, Funcionarios del Cuerpo de Auxilio Judicial, Abogacía del Estado, Ilustres Colegios de Procuradores y Procuradores, Abogados, Graduados Sociales, Administrador del Colegio de Procuradores y, en su caso, del Colegio de Abogados, Letrados de las Cortes Generales y de las Asambleas Legislativas, Funcionarios y Letrados del Servicio Jurídico de la Administración de la Seguridad Social, de las demás Administraciones públicas, de las Comunidades Autónomas o de los Entes Locales, Órganos de la Administración General del Estado, de las Comunidades Autónomas y de las Entidades locales y sus organismos públicos y las Fuerzas y Cuerpos de Seguridad, Administradores concursales, otros que pudieran incluirse mediante la celebración del correspondiente convenio, órganos judiciales y Oficinas de Registro y Reparto. 
- La realización de actos de comunicación procesal conforme a los requisitos establecidos en las leyes procesales.

- La expedición de resguardos electrónicos, integrables en las aplicaciones de gestión procesal, acreditativos de la correcta realización de la presentación de escritos y documentos anexos, de los traslados de copias y de la remisión y recepción de los actos de comunicación procesal y, en todo caso, de la fecha y hora de la efectiva realización.

La constancia de un asiento por cada una de las transacciones electrónicas a que se refieren los números anteriores, realizadas a través del sistema, identificando cada transacción los siguientes datos: identidad del remitente y del destinatario de cada mensaje, fecha y hora de su efectiva realización proporcionada por el sistema y, en su caso, proceso judicial al que se refiere, indicando tipo de procedimiento, número y ańo.

Lexnet, tal y como su nombre indica (la Ley en la Red), pretende extender gran parte de los servicios que ofrece la Administración de Justicia a través de Internet, haciendo que estos sean accesibles desde cualquier parte y en cualquier momento. En la actualidad el sistema permite: el intercambio seguro de información, el uso de firma electrónica reconocida, acceso vía web, funcionamiento $24 \times 7$, ahorro de papel e inmediatez en las comunicaciones.

En las Comunidades Autónomas sin competencias en materia de Justicia o en aquellas que lo han solicitado, esta comunicación se realizará a través del sistema Lexnet, mientras que en el resto se utilizarán los distintos sistemas implantados por cada Comunidad ${ }^{24}$.

${ }^{24}$ Seguidamente se detallan las Comunidades Autónomas que han asumido la administración de Lexnet, en el marco de los convenios de cooperación tecnológica suscritos:

- R Justicia 10 Septiembre 2002 (Convenio entre el M. ${ }^{\circ}$ de Justicia y la Xunta de Galicia para el desarrollo de las nuevas tecnologías de la información y las comunicaciones en la Administración de Justicia) BOE 03/10/2002

- Res.Justicia 2 Junio 2006 (Convenio de cooperación tecnológica con el Departamento de Justicia de la Generalidad de Cataluña para la presentación telemática de escritos y notificaciones en las oficinas judiciales de Cataluña) BOE 26/06/2006

- Res. Justicia 10 Enero 2007 (Convenio de cooperación tecnológica para la implantación y ejecución de la presentación de escritos y notificaciones certificados por vía telemática en los órganos judiciales de la CA Galicia) BOE 02/02/2007 
La implantación de las comunicaciones electrónicas en la administración de Justicia, pasa, según el Ministerio de Justicia, por tres fases dentro de las cuales nos encontramos plenamente inmersos en la actualidad:

- A partir del 1 de enero de 2016: los profesionales de la Justicia (procuradores, abogados, graduados sociales y profesionales de los servicios jurídicos de las administraciones públicas) y todos los órganos y oficinas judiciales y fiscales estarán obligados a emplear los sistemas telemáticos existentes para la presentación de escritos y documentos y para la realización de actos de comunicación (citaciones, notificaciones, requerimientos y emplazamientos) en la totalidad de los órganos jurisdiccionales (civil, penal, social y contencioso-administrativo) para los procedimientos que se inicien a partir de esta fecha, como establece la Ley 42/2015 de 5 de octubre de reforma de la Ley de Enjuiciamiento Civil.

- A partir del 7 de julio de 2016: todas las administraciones con competencias en materia de Justicia estarán obligadas a realizar

- Res. Justicia 15 Abril 2010 (convenio de colaboración entre el Ministerio de Justicia y la Consejería de la Presidencia de la Junta de Castilla y León, para la utilización del sistema LEXNET) BOE 26/04/2010.

- Res. Justicia 16 Noviembre 2010 (Convenio con la Junta de Comunidades de Castilla-La Mancha para establecer un marco de colaboración que favorezca las relaciones con los órganos judiciales mediante la utilización del "sistema Lexnet") BOE 26/11/2010

Res. Justicia 26 Mayo 2011 (Convenio de colaboración con la Comunidad de Madrid para la cesión de uso de derechos del sistema Lexnet del Ministerio de Justicia) BOE 06/06/2011

Res. Justicia 9 Diciembre 2011 (Convenio de colaboración con la CA Canarias para la cesión de los derechos de uso del sistema informático de telecomunicaciones Lexnet) BOE 23/12/2011

- Dictamen 11/2013 de 13 Sep. CA Cataluńa (sobre el RD 396/2013 de 7 Junio, regula el Comité técnico estatal de la Administración judicial electrónica) DOGC 11/10/2013

- Res. Justicia 9 Julio. 2014 (Convenio de colaboración con la Junta de Andalucía, para el desarrollo de actuaciones en el ámbito tecnológico y organizativo en la Administración de Justicia) BOE 23/07/2014

Acuerdo Hacienda y Administraciones Públicas 5 Diciembre 2014 CA Valencia (aprueba la agenda digital de la Comunitat Valenciana 2014-2020) DOCM 09/12/2014

Res. Justicia 26 Diciembre 2014 (Convenio de colaboración con el Gobierno de Canarias para el desarrollo de actuaciones en el ámbito tecnológico y organizativo en la Administración de Justicia) BOE 10/01/2015 
la tramitación electrónica de los procedimientos judiciales como establece la Ley 18/11 de 5 julio reguladora del uso de las tecnologías de la comunicación y la información en la Administración de Justicia.

- A partir del 1 de enero de 2017: la obligatoriedad de utilizar medios electrónicos con la Administración de Justicia se extenderá a las administraciones y organismos públicos y a las Fuerzas y Cuerpos de Seguridad del Estado. También, a las personas que, sin representación o asistencia por un profesional de la Justicia, estén obligadas a comunicarse a través de medios electrónicos con la Administración de Justicia. Además, a partir de esta fecha, los ciudadanos podrán ejercer su derecho a relacionarse _elepáticamente con esta administración cuando no sea perceptiva la intervención de un profesional.

\subsection{SUBASTAS ELECTRÓNICAS}

La Ley 19/2015, de 13 de julio, de medidas de reforma administrativa en el ámbito de la Administración de Justicia y del Registro Civil implanta un nuevo sistema ${ }^{25}$ de subasta electrónica de bienes muebles, inmuebles y bienes hipotecados basado en los principios de publicidad y seguridad.

La subasta se realiza en el Portal de la Agencia Estatal del Boletín Oficial del Estado a cuyo sistema tienen acceso las oficinas judiciales identificando específicamente cada subasta con un número una vez que hayan transcurrido veinticuatro horas desde la publicación del anuncio en el Boletín Oficial del Estado. Se publicará información sobre el bien subastado junto con la documentación oficial correspondiente.

Los interesados en participar en la subasta se registran y utilizan la firma electrónica para identificarse inequívocamente y realizar las respectivas posturas durante un plazo de 20 días a cualquier hora y en cualquier lugar. El letrado de la Administración de Justicia supervisará que el procedimien-

${ }^{25}$ El sistema, avalado por el Consejo General del Poder Judicial, se implantó en 2006 en el Juzgado número 11 de Murcia para permitir la participación en subastas a través de Internet. 
to se lleve a cabo correctamente y finalmente el Portal publicará la puja más alta entre la recibidas.

El Real Decreto 1011/2015, de 6 de noviembre establece un sistema único de constitución telemática de los depósitos necesarios para participar en las subastas y regula la intervención de la Agencia Tributaria para gestionar las pujas e ingresar en la Cuenta de Depósitos y Consignaciones del Juzgado la puja ganadora de la subasta.

Aún no se disponen de datos estadísticos oficiales sobre el éxito de la celebración de este tipo de subastas si bien se puede destacar que descargará de trabajo a los juzgados y abrirá a la ciudadanía una fórmula transparente, sencilla y ágil para participar en las subastas.

\section{CONCLUSIONES}

Los sucesivos planes para la modernización de la justicia constituyen un ambicioso proyecto que pretende renovar un sistema de justicia antiguo, lento y poco flexible a los cambios, que se mostró incapaz de lidiar con los nuevos retos de los sistemas de justicia de las sociedades avanzadas. Con los cambios que se están instaurando se pretende combatir los problemas organizativos y los defectos estructurales de un sistema caduco desbordado por los cambios sociales, la falta de medios humanos, técnicos y materiales, una legislación deficiente en términos operativos y procesales y una creciente juridificación de la vida social.

Nos encontramos ante un proceso sumamente complicado, debido a la complejidad del sistema y el elevado número de actores e instituciones que participan en el mismo. Además, en el ámbito de la Administración de Justicia confluyen responsabilidades tanto del Consejo General del Poder Judicial, como del Ministerio de Justicia y de las Comunidades Autónomas con competencias transferidas en esta materia. Por eso la implantación y desarrollo de todas estas medidas se está realizando con distinto grado de intensidad y eficacia en las distintas provincias.

Las mejoras que se están operando parten de la base de un cambio de paradigma organizativo, procesal, de gestión y también tecnológico. Y la efectiva implantación de una Justicia profesional, ágil, clara, transparente, 
confiable, predecible, moderna, eficaz y que sea accesible para los ciudadanos requiere reformas en profundidad que ya se están acometiendo.

Actualmente se implementan cambios importantes con las novedades que supone el despliegue de todas las medidas que se han establecido junto con cambios normativos que irán llegando en los próximos meses. En el presente trabajo nos hemos referido a las más importantes reformas en el ámbito del derecho procesal civil como la presentación de escritos por vía telemática, las formas de realizar los actos de notificación, las subastas electrónicas, etc. Y la realidad es que se están consiguiendo importantes avances con la implantación de las nuevas tecnologías. Lo que concluimos con este artículo es que todos estos cambios son condición imprescindible, pero no suficiente, para la enorme labor que supone agilizar la administración de justicia española, porque desde luego que la tecnología, por sí sola, no puede sostener un cambio como el que se necesita, y es preciso que este impulso modernizador venga acompańado de una batería de medidas en otros ámbitos. Desde luego que un Plan de Modernización como el que estamos viviendo no tiene precedentes en nuestro país, tampoco el aumento del presupuesto asignado a tal fin, que ha aumentado en más de un $50 \%$ en los últimos ańos, evidenciando una clara voluntad política de llevar a cabo medidas destinadas a crear un sistema de justicia adecuado a las necesidades actuales y futuras y a criterios de racionalización, optimización y eficiencia. Empezamos a ver ya los efectos de todas las reformas acometidas, aunque en unos dos años los resultados serán más evidentes y podremos realizar una valoración cualitativa y cuantitativa de lo que ha supuesto esta mejora y modernización de la administración de justicia en España.

\section{REFERENCES:}

Bueno de Mata, Federico. 2014. Prueba electrónica y proceso 2.0, Tirant lo Blanch, Valencia;

Cabezudo Rodríguez, Nicolás "La administración de Justicia ante las innovaciones tecnológicas. Del entusiasmo a la desconfianza pasando por el olvido legal”. Revista Jurídica de Castilla y León, no 7, León, 2005; 
Gascón Inchausti, Fmando. 2010. "La e-justicia en la Unión Europea: Balance de la situación y planes para el futuro en diciembre de 2009 en Carmen Senés Montilla (coord.) Presente y futuro de la e-Justicia en España y en la Unión Europea, Thompson Reuters - Aranzadi, Pamplona;

Garrido Gómez, Isabel, J. Alberto Del Real Alcalá, Angeles Solanes Corella. 2015. Modernización y mejora de la Administración de Justicia y de la operatividad de los jueces en España. Documentos de Trabajo (IELAT, Instituto de Estudios Latinoamericanos), no 71, p. 1-35;

Martín Pastor, Jose. 2008. "Un paso importante hacia el proceso telemático en Espańa: el sistema informático de telecomunicaciones Lexnet para la presentación de escritos y documentos, el traslado de copias y la realización de actos de comunicación procesal por medios telemáticos”, en CARPI, F. ORTELLS, RAMOS, M. (coord.) Oralidad y escritura en un proceso civil eficiente, Universidad de Valencia, Valencia;

Peiteado Mariscal, Pilar. 2010. Posibles aplicaciones telemáticas en el proceso civil, en Carmen Senés Montilla (coord.) Presente y futuro de la e-Justicia en España y en la Unión Europea, Thompson Reuters- Aranzadi, Pamplona;

Rayón Ballesteros, Maria Concepción. La necesaria modernización de la justicia: especial referencia al plan estratégico 2009-2012. Anuario jurídico y económico escurialense, Madrid, 2011, no 44, p. 173-190. 